\title{
On the Design and Efficient Implementation of the Farrow Structure
}

\author{
Carson K. S. Pun, Student Member, IEEE, Y. C. Wu, Student Member, IEEE, S. C. Chan, Member, IEEE, and \\ K. L. Ho, Member, IEEE
}

\begin{abstract}
This letter proposes an efficient implementation of the Farrow structure using sum-of-powers-of-two (SOPOT) coefficients and multiplier-block (MB). In particular, a novel algorithm for designing the Farrow coefficients in SOPOT form is detailed. Using the SOPOT coefficient representation, coefficient multiplication can be implemented with limited number of shifts and additions. Using MB, the redundancy between multipliers can be fully exploited through the reuse of the intermediate results generated. Design examples show that the proposed method can greatly reduce the complexity of the Farrow structure while providing comparable phase and amplitude responses.
\end{abstract}

Index Terms-Farrow structure, fractional-delay digital filters, multiplier-block (MB), sum-of-powers-of-two (SOPOT).

\section{INTRODUCTION}

$\mathbf{F}$ RACTIONAL-DELAY digital filters (FD-DF) are very useful in providing fractional delay of digital signals. It finds important applications in software radio [1], digital modems [2], arbitrary sampling rate conversion, time-delay estimation [3], etc. In general, the fractional-delay digital filters can be implemented either as finite-duration impulse response (FIR) or infinite-duration impulse response (IIR) digital filters [7]. FIR-based FD-DFs are usually implemented by the Farrow structure [4] because it can provide variable signal delay, making high-speed online tuning feasible. The implementation of IIR-based FD-DFs is, however, rather complicated due to the stability constraints and transient problem. Also, to avoid the implementation of polynomial fractions, approximate realization such as the gathering structure [12] has to be used. Because of these reasons, we only consider FIR-based FD-DFs.

In this letter, an efficient implementation of the Farrow structure using sum-of-powers-of-two (SOPOT) coefficients and multiplier-block (MB) is proposed. Furthermore, a novel algorithm for determining the Farrow coefficients with SOPOT representation is also presented. More precisely, the coefficients of the subfilters in the Farrow structure are represented as SOPOT coefficient or canonical signed digits (CSDs). The SOPOT coefficient is attractive for very large scale integration or hardware implementation because multiplication of SOPOT coefficients can be implemented efficiently using

Manuscript received August 16, 2001; revised October 4, 2002. The work was supported by a grant from the Research Grants Council of the Hong Kong SAR. The associate editor coordinating the review of this manuscript and approving it for publication was Dr. Elias S. Manolakos.

The authors are with the Department of Electrical and Electronic Engineering The University of Hong Kong (e-mail: carson@ieee.org; ycwu@eee.hku.hk; scchan@eee.hku.hk; klho@eee.hku.hk).

Digital Object Identifier 10.1109/LSP.2003.813681 limited number of hard-wired shifters and adders only (i.e., multiplierless). To further reduce the number of adders required in this structure, the subfilters in the Farrow structure are implemented in transposed form, which allows us to implement all the SOPOT multiplications with a single MB [5], [6]. MB is an efficient technique for reducing the number of additions in multiplying a variable input with a fixed set of coefficients in binary or CSD representations. The basic principle of the MB is to reuse the intermediate results generated in realizing some of the coefficients in order to reduce the adders or additions required for the other coefficients. For example, if there are two SOPOT coefficients "7" and " 21 " to be multiplied by the input signal $x$, the direct implementation will require three adders, since " $7 x$ " equals " $(8-1) x$," and " $21 x$ " equals " $(18+2+1) x$ " in SOPOT form. However, if the multiplications are implemented together in an MB , the number " 21 " will be rewritten as " $7 \times(2+1)$." Therefore, the result of $21 x$ can be realized by first computing the result " $y=7 x$ " and then multiplying it with " $3=(2+1)$." By so doing, only two adders are required in the $\mathrm{MB}$ as a result of reusing the intermediate value $y$. MB has been proposed to implement interpolated filter and filter banks [5]. Unfortunately, detailed design of such multiplierless Farrow structure was not addressed. This motivates the proposed design and implementation methods of the Farrow structure.

The proposed algorithm consists of two different steps: an FD-DF in Farrow structure with real-valued coefficients is first designed using any conventional methods [4], [7], [10]; a flexible and efficient "random search" algorithm is then employed to search for the SOPOT coefficients around the real-valued coefficients while minimizing some criteria such as the number of SOPOT terms used subject to a given frequency specification. Design results show that significant saving in hardware complexity can be obtained by the proposed method.

\section{EFFicIEnT FarRow Structure With SOPOT COEFFICIENTS AND MB}

The output of the FD-DF $y[(m+D+\mu) T]$ is given by

$$
y[(m+D+\mu) T]=\sum_{i=0}^{N-1} x[(m-i) T] \cdot h_{\mu}(i)
$$

where $x[m T]$ is the input signal sampled at a period $T ; h_{\mu}(i)$ is the impulse response of the FD-DF with a system delay of $D+\mu$, where $D$ is an integer constant, and $\mu$ is the fractional 


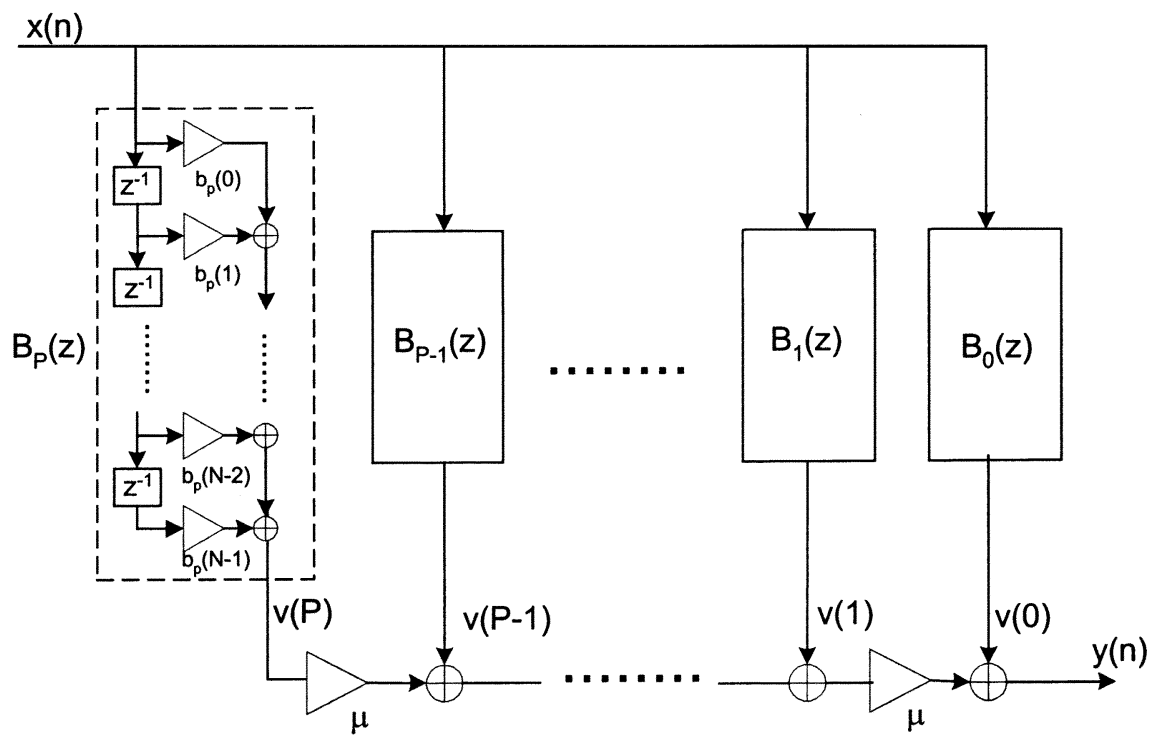

Fig. 1. Original Farrow structure.

delay parameter between zero and one. $N$ is the length of $h_{\mu}(i)$. To avoid the implementation of a large number of filters with different delays, Farrow [4] proposed to approximate each impulse response $h_{\mu}(i)$ with the following $P$ th-order polynomial in delay value $\mu$ such that the delay control is independent of the filter coefficients

$$
h_{\mu}(i)=\sum_{n=0}^{P} b_{n}(i) \mu^{n}
$$

Substituting (2) into (1) gives

$$
y[(m+D+\mu) T]=\sum_{n=0}^{P}\left[\sum_{i=0}^{N-1} x[(m-i) T] \cdot b_{n}(i)\right] \mu^{n} .
$$

Fig. 1 shows the Farrow structure for implementing (3), where the input signal is passed through a number of subfilters $b_{n}(i)$, $n=0, \ldots, P$, and is multiplied by the appropriate powers of $\mu$ to produce the output.

Though the Farrow structure is very useful in providing a continuous value of signal delay for digital signals by changing the value of $\mu$, it still requires large number of multiplications for the subfilter implementation, especially when $P$ and $N$ are large to provide very precise control of the frequency characteristics of the FD-DF. One method to avoid the expensive multipliers is to convert the Farrow coefficients $b_{n}(i)$, which is fixed, into the following SOPOT representation:

$$
\hat{b}_{n}(i)=\sum_{k=1}^{L} b_{n, k}(i) \cdot 2^{a_{k}}
$$

with $b_{n, k}(i) \in\{-1,1\}$ and $a_{k} \in\{-l, \ldots,-1,0,1, \ldots, l\}$, where $l$ is a positive integer, and its value determines the range of the coefficients. $L$ is the number of terms used in the coefficient approximation and is usually limited to a small number. The coefficient multiplications can therefore be implemented as limited shifts and additions, resulting in a significant reduction in implementation complexity. Very often, there is also significant redundancy in these SOPOT coefficients, which appears as common subexpressions among different SOPOT coefficients. Due to the $z$ operator, it is somewhat difficult to remove these subexpressions. Fortunately, thanks to the transposed form of the subfilters, the Farrow structure can be rewritten as in Fig. 2. In this new structure, the input is multiplied to a number of constant coefficients. Hence, the common subexpressions within the SOPOT coefficients can be eliminated [8], [9] using a single $\mathrm{MB}$, which further reduces the complexity of the Farrow structure. Next, we shall consider the problem of determining the Farrow coefficients in SOPOT form.

\section{SOPOT COEFFICIENTS DESIGN AND MB GENERATION}

Let $H\left(e^{j \omega}, \mu\right)=e^{j \omega \mu}$ and $\hat{H}\left(e^{j \omega}, \mu\right)$ be the frequency responses of the ideal fractional delay filter and that of the Farrow structure with SOPOT coefficients. Our objective is to determine the SOPOT coefficients $\hat{b}_{n}(i)$ such that the distortion from the ideal response is minimal. Commonly used distortion measures such as the least squares and the minimax criterion can be used. Without loss of generality, the minimax criterion is employed and the problem can be stated as follows.

Given the maximum number of terms $L$ in each coefficient, the dynamic range $l$ of the coefficients and the bandwidth of interest $\left(0, \omega_{p}\right)$, determine the SOPOT coefficients $\hat{b}_{n}(i)$ such that the maximum phase response error $\delta_{p}$ is minimized subject to a constraint that the peak amplitude error $\delta_{a}<\varepsilon$, where

$$
\begin{aligned}
& \delta_{p}=\max _{0<\omega<\omega_{P},|\mu|<0.5}\left[\left|\frac{\omega \mu-\arg \left\{\hat{H}\left(e^{j \omega}, \mu\right)\right\}}{\omega}\right|\right] \\
& \delta_{a}=\max _{0<\omega<\omega_{P},|\mu|<0.5}\left[|1-| \hat{H}\left(e^{j \omega}, \mu\right)||\right] .
\end{aligned}
$$

Let $\boldsymbol{b}_{I}$ be the vector containing the real-valued coefficients of the Farrow structure $b_{n}(i)$ designed by using any conventional FD-DF design method and interpolation. The principle of the 


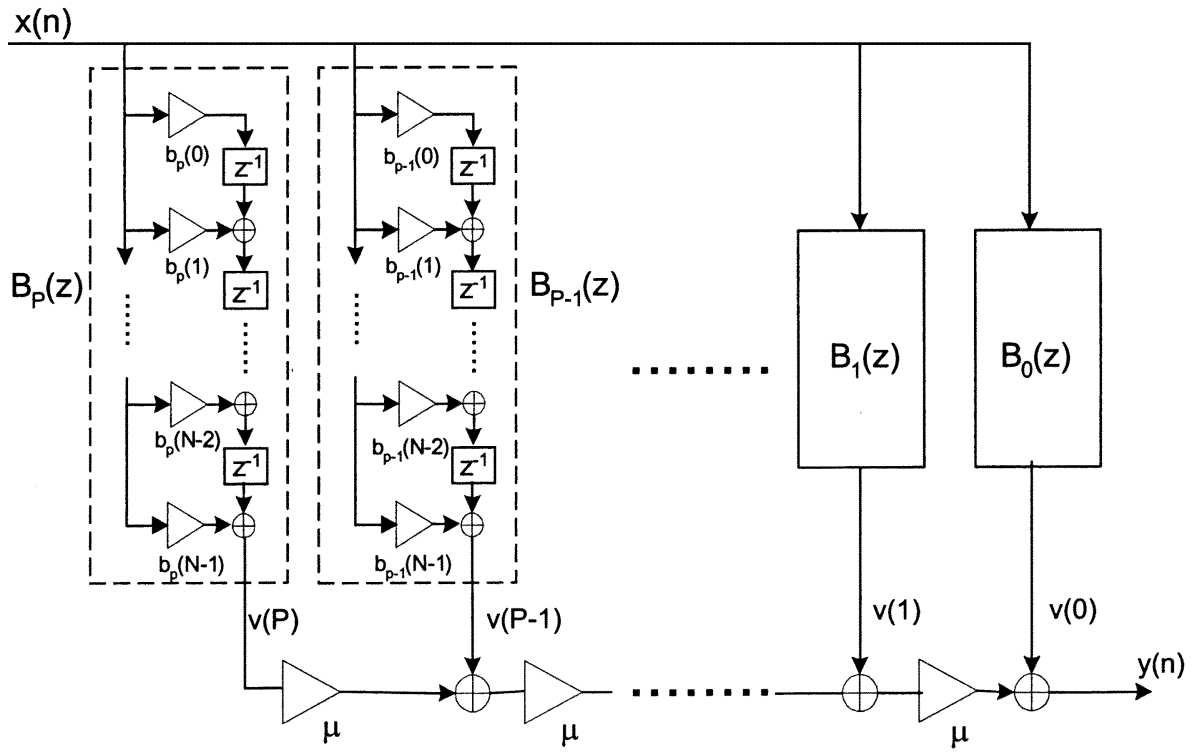

Fig. 2. Proposed implementation of the Farrow structure.

proposed random search algorithm is to generate random candidate SOPOT coefficients in the neighborhood of $\boldsymbol{b}_{I}$ so as to search for the optimal discrete solution. More precisely, a new coefficient vector $\boldsymbol{b}_{\mathrm{NEW}}$ is generated by adding a random vector to the original coefficient vector $\boldsymbol{b}_{I}$ as follows:

$$
\boldsymbol{b}_{\mathrm{NEW}}=\left\lfloor\boldsymbol{b}_{I}+\alpha \cdot \boldsymbol{b}_{R}\right\rfloor_{\mathrm{SOPOT}}
$$

where $\alpha$ is a scale factor that controls the size of neighborhood to be searched; $\boldsymbol{b}_{R}$ is a vector with its elements being random numbers in the range $[-1,1]$; and $\lfloor\cdot\rfloor_{\text {SOPOT }}$ is the rounding operation that convert its argument to the nearest SOPOT coefficients with maximum number of terms in each coefficient being $L$ and dynamic range $l$. The performance measures $\delta_{p}$ and $\delta_{a}$ of the new coefficients are then calculated. The set that yields the minimum phase error $\delta_{p}$ with $\delta_{a}$ smaller than a given peak amplitude error $\varepsilon$ is the optimum solution under the constraints of $L$ and $l$. As this is a random search algorithm, the longer the searching time, the higher the chance of finding the optimal solution.

After the SOPOT representation is obtained, the minimum number of adders needed in the MB is determined using the algorithm proposed in [6]. Due to space limitation, the detail is not included here. Interested readers can find more detail in [6].

This random search algorithm is similar to the mutation of genetic algorithm and the random walk in stimulated annealing [13]. The main difference here is that we have limited its search space to a small neighborhood of the real-valued solution. This greatly shortens the search time to a few minutes. In fact, for the problem considered here, the overall design time only takes less than 5 min to complete on a typical Pentium-400 PC using Matlab 5.3., including both the design of SOPOT coefficients and the MB design. Another advantage of this algorithm is that it can also be used to minimize directly the hardware cost such as adder cells of the filters, taking into account round-off and overflow noise [11]. Moreover, a set of possible solutions representing different tradeoffs between computational complexity
TABLE I

OPTIMIZED SOPOT COEFFICIENTS IN EXAMPLE 1

\begin{tabular}{c|c|c|c|c}
\hline$n$ & $b_{3}(n)$ & $b_{2}(n)$ & $b_{1}(n)$ & $b_{0}(n)$ \\
\hline 0 & $-2^{-6}+2^{-8}$ & $2^{-4}-2^{-7}$ & 0 & $-2^{-6}+2^{-10}$ \\
\hline 1 & $2^{-4}+2^{-6}-2^{-8}$ & $-2^{-2}+2^{-5}+2^{-8}$ & $-2^{-6}$ & $2^{-4}-2^{-7}$ \\
\hline 2 & $-2^{-1}+2^{-3}-2^{-5}$ & $2^{-1}+2^{-3}+2^{-7}$ & $2^{-3}-2^{-5}+2^{-8}$ & $-2^{-3}-2^{-5}-2^{-9}$ \\
\hline 3 & $2^{-0}-2^{-3}+2^{-5}$ & $-2^{-1}+2^{-5}+2^{-8}$ & $-2^{-0}-2^{-2}+2^{-5}$ & $2^{-1}+2^{-3}-2^{-7}$ \\
\hline 4 & $-2^{-0}+2^{-3}-2^{-5}$ & $-2^{-1}+2^{-5}+2^{-8}$ & $2^{-0}+2^{-2}-2^{-5}$ & $2^{-1}+2^{-3}-2^{-7}$ \\
\hline 5 & $2^{-1}-2^{-3}+2^{-5}$ & $2^{-1}+2^{-3}+2^{-7}$ & $-2^{-3}+2^{-5}-2^{-8}$ & $-2^{-3}-2^{-5}-2^{-9}$ \\
\hline 6 & $-2^{-4}-2^{-6}+2^{-8}$ & $-2^{-2}+2^{-5}+2^{-8}$ & $2^{-6}$ & $2^{-4}-2^{-7}$ \\
\hline 7 & $2^{-6}-2^{-8}$ & $2^{-4}-2^{-7}$ & 0 & $-2^{-6}+2^{-10}$ \\
\hline
\end{tabular}

and performance will be generated during the search. Therefore, it helps one to achieve an appropriate tradeoff for a given application. It is also possible to combine the two stages together to improve the performance, but the computational time will be greatly increased. Finally, it should be noted that the proposed design and realization methods are also applicable to the multiplierless realization of the gathering structure recently proposed in [12] for implementing IIR FD-DFs.

\section{EXAMPLES}

Example 1: Let us consider the coefficients provided by Farrow in [4]. The bandwidth under consideration for this filter is from 0 to $0.6 \pi$. $\varepsilon$ is set to 0.006 . The interpolation order is three, and the length of each FIR filters used in the Farrow structure is eight. The value of " $l$ " in (4) is chosen to be ten. The optimized SOPOT coefficients of the Farrow structure are given in Table I. For the Farrow structure with real-valued coefficients, the peak ripple error and maximum phase delay error deviated from the ideal frequency response are 0.006271 and 0.0032, respectively; whereas for the SOPOT Farrow structure, $\delta_{a}$ and $\delta_{p}$ are found to be 0.005371 and 0.0046 , respectively. This shows that a comparable performance can be achieved using SOPOT coefficients. After common-subexpressions elimination, the MB requires only 13 adders, which compares favorably with 32 real multiplications in the original Farrow 

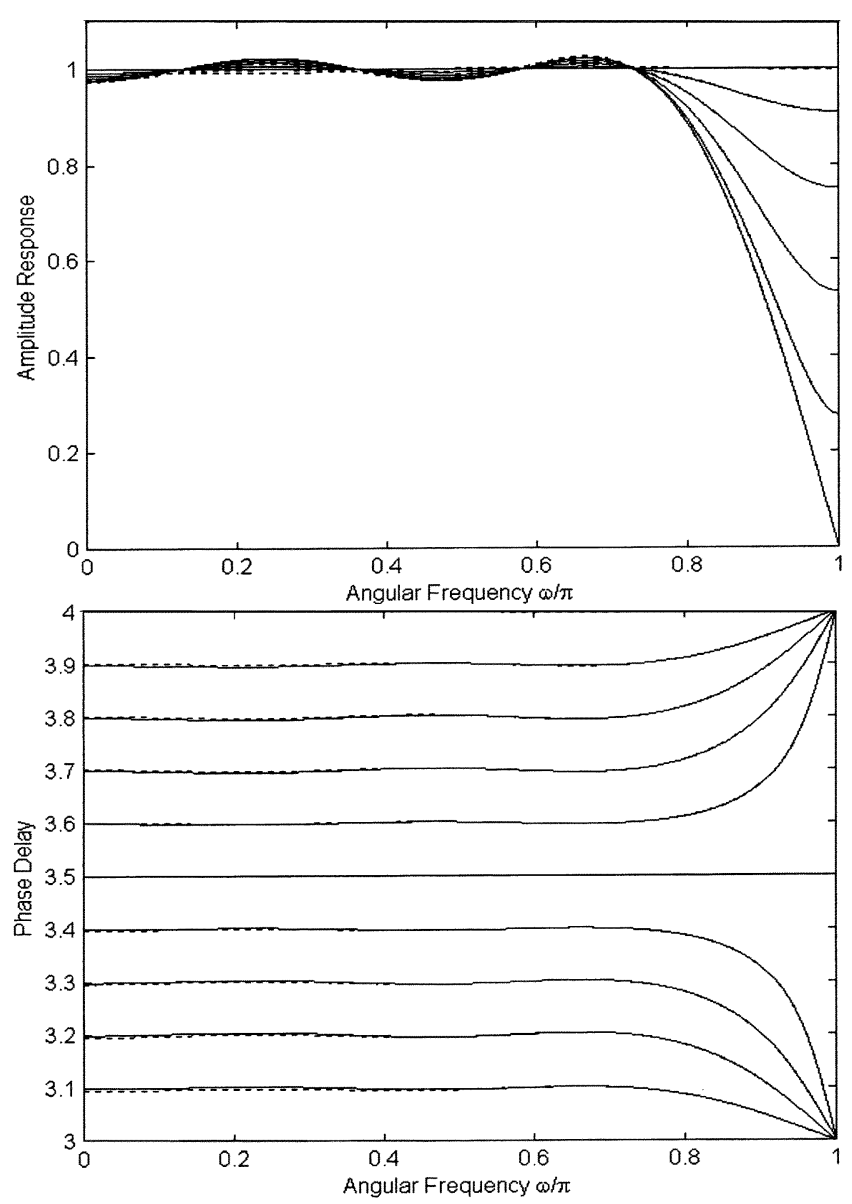

Fig. 3. Comparison of amplitude and phase responses between the proposed structure (dotted line) and the original Farrow structure with real-valued coefficients (solid line) in Example 2.

structure. The resultant Farrow structure filter has a much lower complexity than the real-valued Farrow structure but providing nearly the same phase delay and amplitude response.

Example 2: This example will be on a Farrow structure with higher polynomial order. Although there are many methods for designing FD-DF with real-valued coefficients [4], [7], [10], the prototype fractional delay filters in this example are designed using complex Chebyshev approximation, because it is readily available in MATLAB. They are then interpolated by a fifth-order polynomial to obtain the real-valued Farrow coefficients. The length of each FIR filter in the Farrow structure is again chosen to be eight, and the value of " $l$ " is ten. The bandwidth under consideration is from 0 to $0.75 \pi$. $\varepsilon$ is set to 0.03 . The peak ripple error and maximum phase delay error of the original Farrow structure are 0.023256 and 0.0054 , respectively. After SOPOT coefficients searching and common-expression elimination, the subfilters require only 18 adders to achieve $\delta_{a}=0.026376$ and $\delta_{p}=0.0059$. In Fig. 3, the frequency responses of the proposed structure (dotted lines) and its real-values counterpart (solid lines) are compared for different values of $\mu$. It can be seen that they are very close to each other. The performance and arithmetic complexity of the two examples are summarized in Table II. Again, significant reduction in hardware complexity by the proposed method is observed.
TABLE II

SUMMARY OF DESIGN EXAMPLES

\begin{tabular}{l|c|c}
\hline & Ex 1. & Ex 2. \\
\hline Original peak ripple error & 0.006271 & 0.023256 \\
\hline Original max. phase delay error & 0.0032 & 0.0054 \\
\hline $\begin{array}{l}\text { Peak ripple error using SOPOT } \\
\text { coefficients }\end{array}$ & 0.005371 & 0.026376 \\
\hline $\begin{array}{l}\text { Max. phase delay error using } \\
\text { SOPOT coefficients }\end{array}$ & 0.0046 & 0.0059 \\
\hline Bandwidth of interest & $(0,0.6 \pi)$ & $(0,0.75 \pi)$ \\
\hline $\begin{array}{l}\text { Original number of multipliers } \\
\text { Number of adders using SOPOT } \\
\text { coefficients }\end{array}$ & 32 & 48 \\
\hline $\begin{array}{l}\text { Number of adders using SOPOT } \\
\text { coefficients and MB }\end{array}$ & 13 & 78 \\
$\begin{array}{l}\text { \% of adders reduction using MB } \\
\text { Design Time used on Pentium-400 } \\
\text { (Minutes) }\end{array}$ & $72.92 \%$ & 4 \\
\hline
\end{tabular}

\section{CONCLUSION}

A new method for the design and efficient implementation of Farrow-based FD-DF using SOPOT coefficients and MB is presented. Considerable saving in hardware complexity can be achieved by the proposed method with a reasonable fast design time and a frequency response comparable to their real-valued counterpart. The usefulness of the method is demonstrated with several design examples.

\section{REFERENCES}

[1] T. Hentschel and G. Fettweis, "Sample rate conversion for software radio," IEEE Commun. Mag., vol. 38, pp. 142-150, Aug. 2000.

[2] L. Erup, F. M. Gardner, and R. A. Harris, "Interpolation in digital modems. II. Implementation and performance," IEEE Trans. Commun., vol. 41, pp. 998-1008, June 1993.

[3] H. C. So, P. C. Ching, and Y. T. Chan, "A new algorithm for explicit adaptation of time delays," IEEE Trans. Signal Processing, vol. 42, pp. 1816-1820, July 1994.

[4] C. W. Farrow, "A continuously variable digital delay element," in Proc. ISCAS, 1988, pp. 2641-2645.

[5] A. G. Dempster and N. P. Murphy, "Efficient interpolators and filter banks using multiplier blocks," IEEE Trans. Signal Processing, vol. 48, pp. 257-261, Jan. 2000.

[6] A. G. Dempster and M. D. Macleod, "Use of minimum-adder multiplier blocks in FIR digital filters," IEEE Trans. Circuits Syst. II, vol. 42, pp. 569-577, Sept. 1995.

[7] T. I. Laakso, V. Valimaki, M. Karjalainen, and U. K. Laine, "Splitting the unit delay, tools for fractional delay filter design," IEEE Signal Processing Mag., pp. 30-60, Jan. 1996.

[8] M. Potkonjak, M. B. Srivastava, and A. P. Chandrakasan, "Multiple constant multiplications: Efficient and versatile framework and algorithms for exploring common subexpression elimination," IEEE Trans. Computer-Aided Design, vol. 15, pp. 151-165, Feb. 1996.

[9] R. Pasko, P. Schaumont, V. Derudder, and D. Durackova, "Optimization method for broadband modem FIR filter design using common subexpression elimination," in Proc. Symp. System Synthesis, 1997, pp. $100-106$.

[10] J. Vesma and T. Saramaki, "Design and properties of polynomial-based fractional delay filters," in Proc. ISCAS, 2000, pp. 104-107.

[11] C. K. S. Pun, S. C. Chan, and K. L. Ho, "Efficient design of a class of multiplier-less perfect reconstruction two-channel filter banks and wavelets with prescribed output accuracy," in Proc. 11th IEEE Workshop Statistical Signal Processing, Singapore, Aug. 2001, pp. 599-602.

[12] M. Makundi, T. I. Laakso, and V. Valimaki, "Efficient tunable IIR and allpass filter structures," Electron. Lett., vol. 37, pp. 344-345, Mar. 2001.

[13] D. Karaboga and D. T. Pham, Intelligent Optimization Techniques: Genetic Algorithms, Tabu Search, Simulated Annealing and Neural Networks. Berlin, Germany: Springer-Verlag, 2000. 